\title{
LA-UR-19-26377
}

Approved for public release; distribution is unlimited.

Title:

From Biowaste to Rocket Fuel

Author(s): $\quad$ Evans, Amanda Christine

Intended for: DisrupTech Presentation

Issued: 
Disclaimer:

Los Alamos National Laboratory, an affirmative action/equal opportunity employer, is operated by Triad National Security, LLC for the National Nuclear Security Administration of U.S. Department of Energy under contract 89233218CNA000001. By approving this article, the publisher recognizes that the U.S. Government retains nonexclusive, royalty-free license to publish or reproduce the published form of this contribution, or to allow others to do so, for U.S. Government purposes. Los Alamos National Laboratory requests that the publisher identify this article as work performed under the auspices of the U.S. Department of Energy. Los Alamos National Laboratory strongly supports academic freedom and a researcher's right to publish; as an institution, however, the Laboratory does not endorse the viewpoint of a publication or guarantee its technical correctness. 


\section{From Biowaste to Rocket Fuel}

Dr. Amanda Evans

Scientist 3

B-11, Biosciences

acevans@lanl.gov

(505) 667-3630 


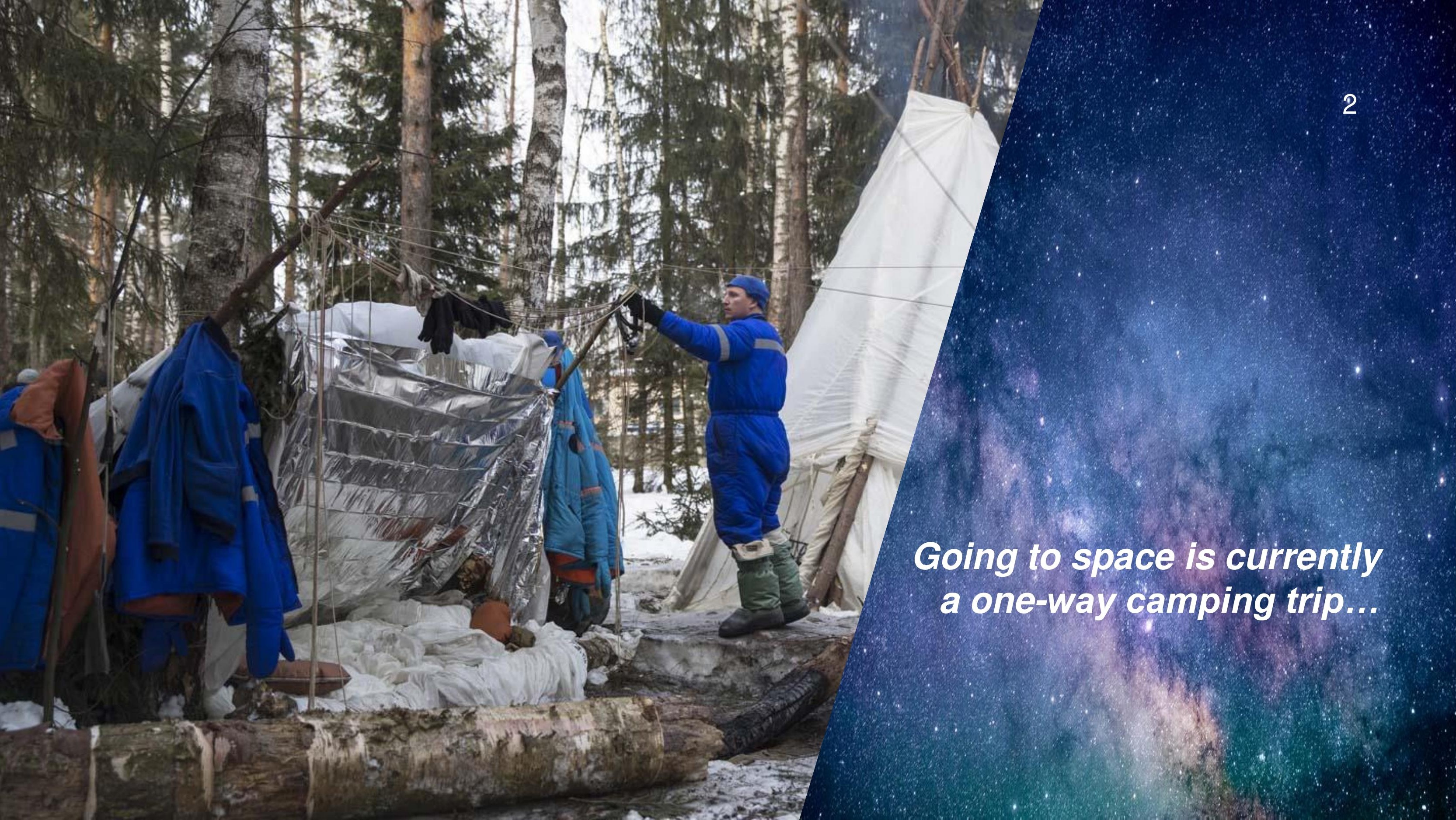


"And if you solve enough problems, you get to come home."

\section{-The Martian}




\section{HYDRAZZINE}

Stanđard Ŗocket Fụel

\section{years}

(2) Highly toxic \& cârcinogenic

(2) Highly flammable

(s) Expensive storage,

i: i... hàndlithg \& disposal

100,000 metric tons manufactured annuailly

Market cost of Ultra Pure Hydrazine $=\$ 85-100 / \mathrm{lb}$.

Safety cost for fueling satellites $=$ $>\$ 100,000 /$ launch 


\section{Green Rocket Fuel}
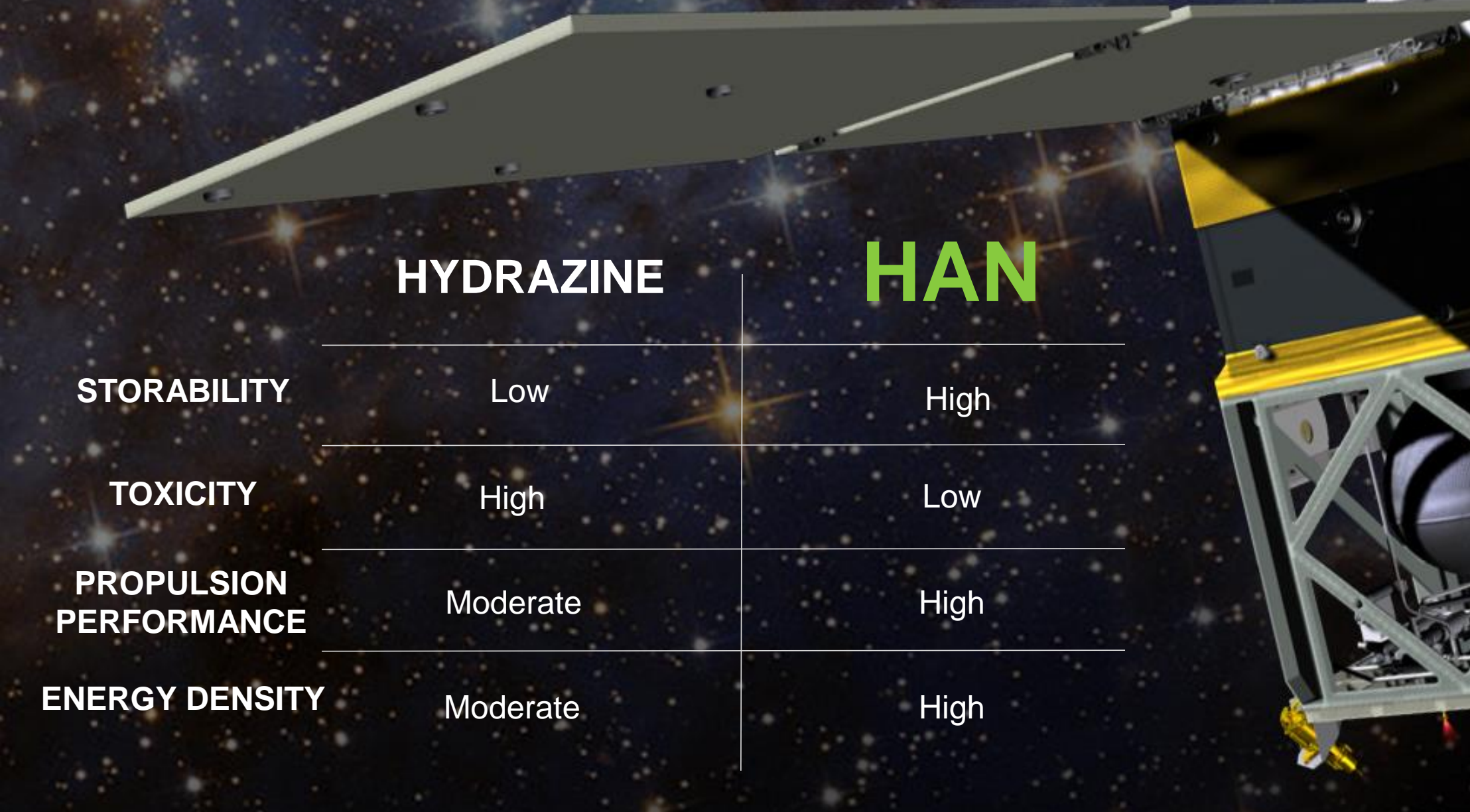

$50 \%$

more
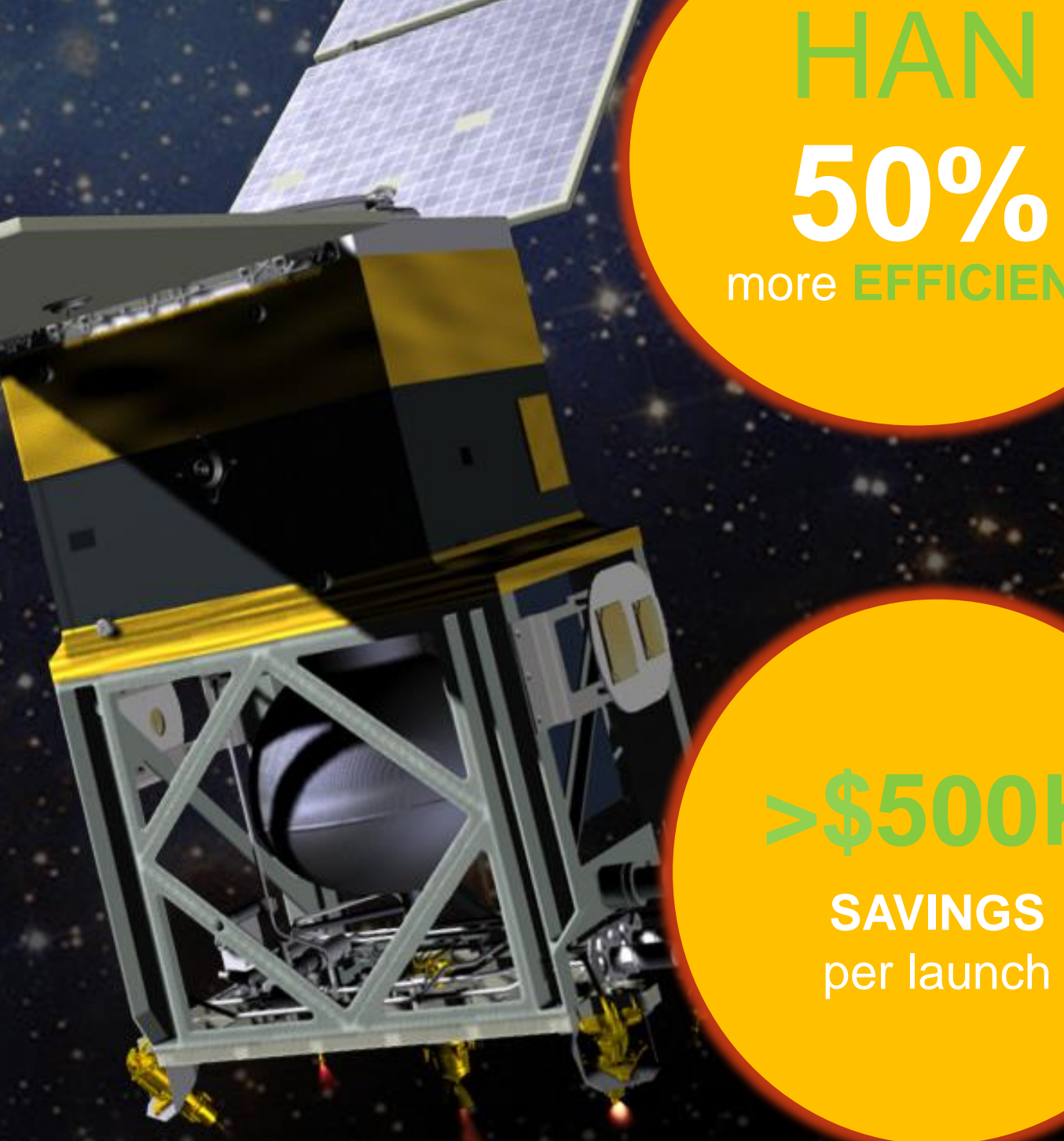

SAVINGS per launch 
HAN and more...
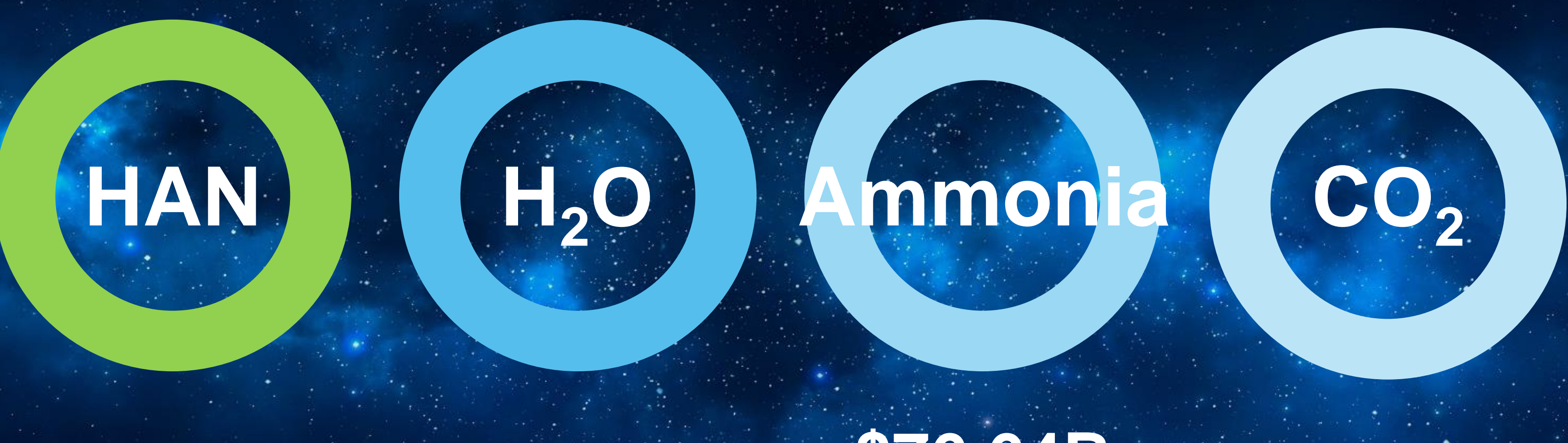

$\$ 76.64 \mathrm{~B}$

by 2025 


\section{Continuous ON-DEMAND manufacturing in space is ESSENTIAL}
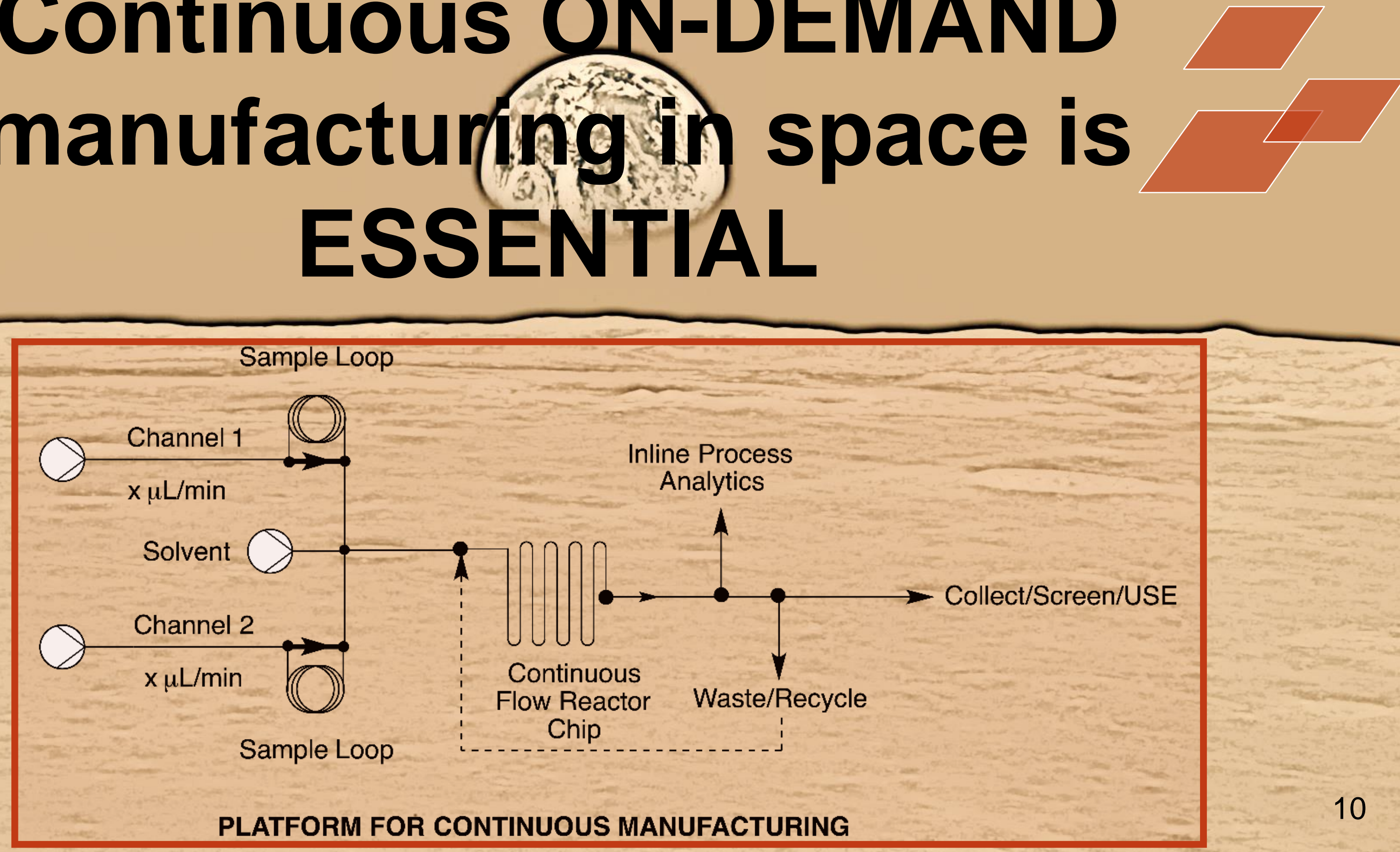


\section{The Process}

Recycled Water as Pročess Solvent

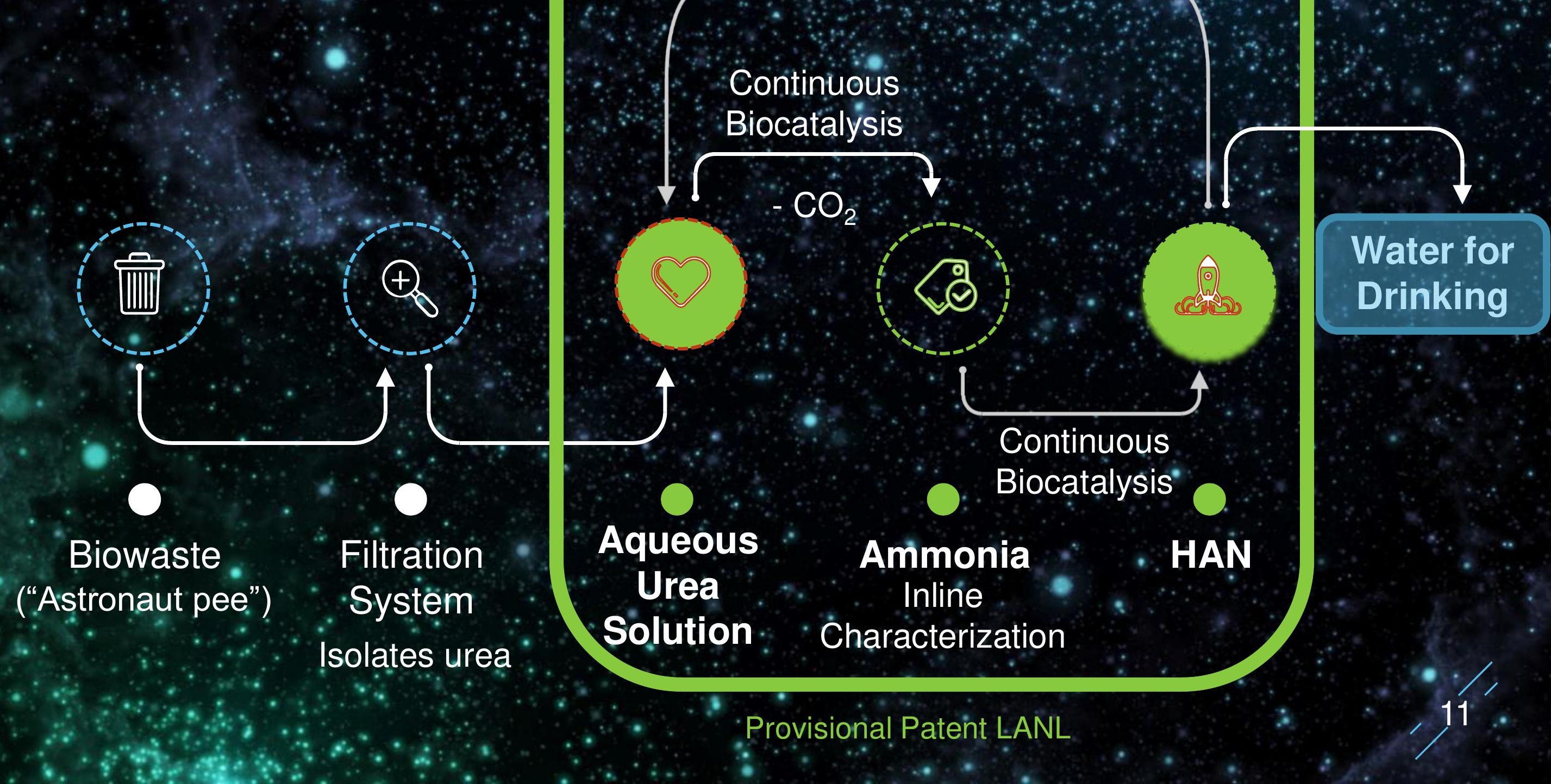


Rocket fuel market :

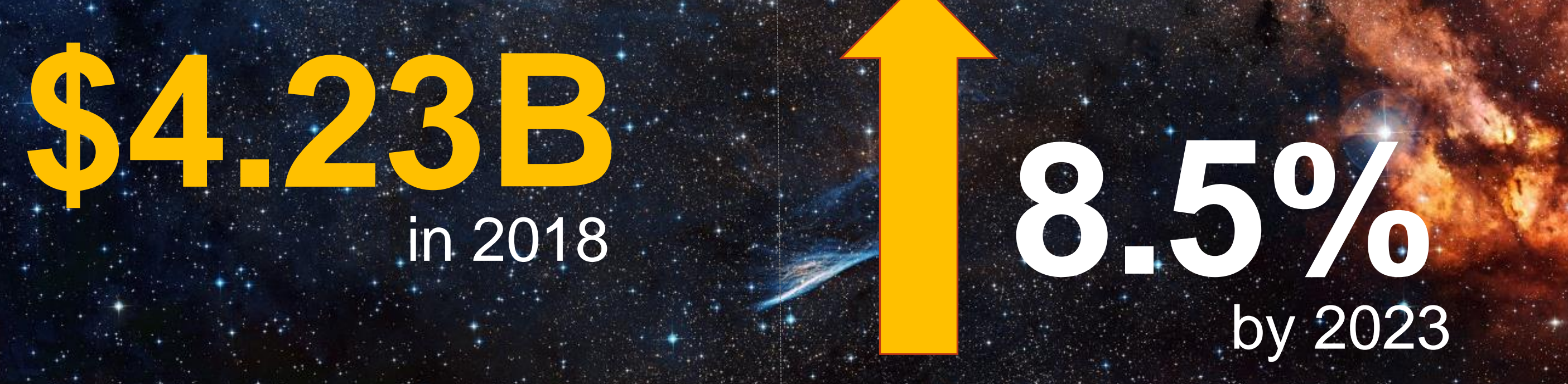


BOCKET FUEL MARKET

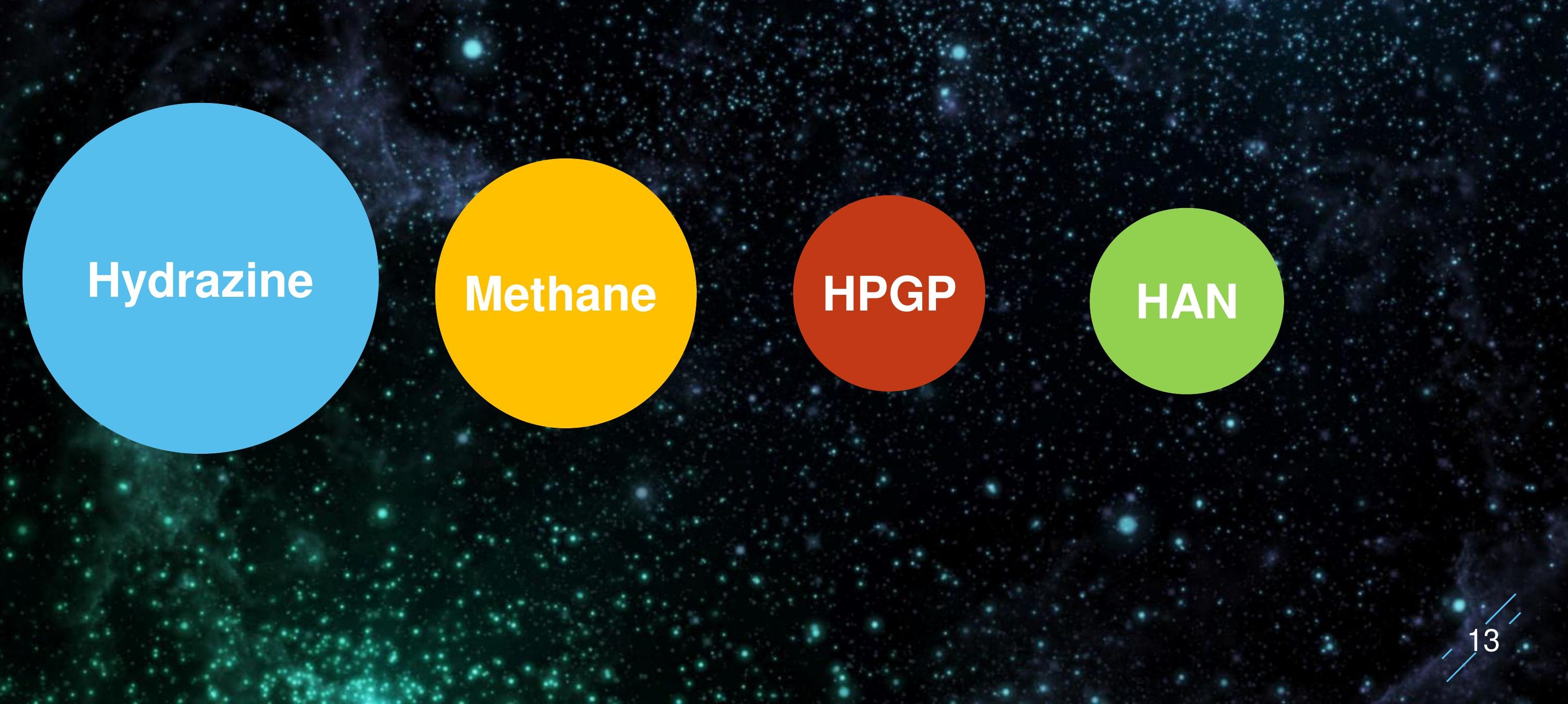




\section{Go to market timeline}

$\mathcal{W}$ ISS National

Lab/Commercial Partner Investment

Microgravity/Space Based TED

$\$ 750,000$ Required
2019

LANL Investment

Ground Based Technical

Evaluation \& Demonstration

(TED)

$\$ 125,000$
2021

2022

NASA Investment

isS Filtration System

Integration TED

of $\$ 1.25$ million required
Commercial Space Investment Cube Satellite TED

$\$ 1.75$ million required

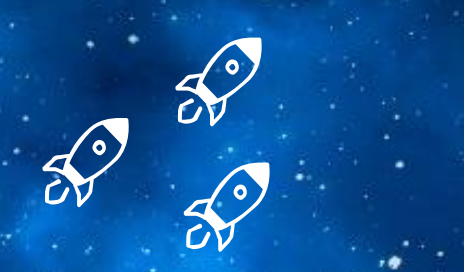




\section{Prospective Partners}

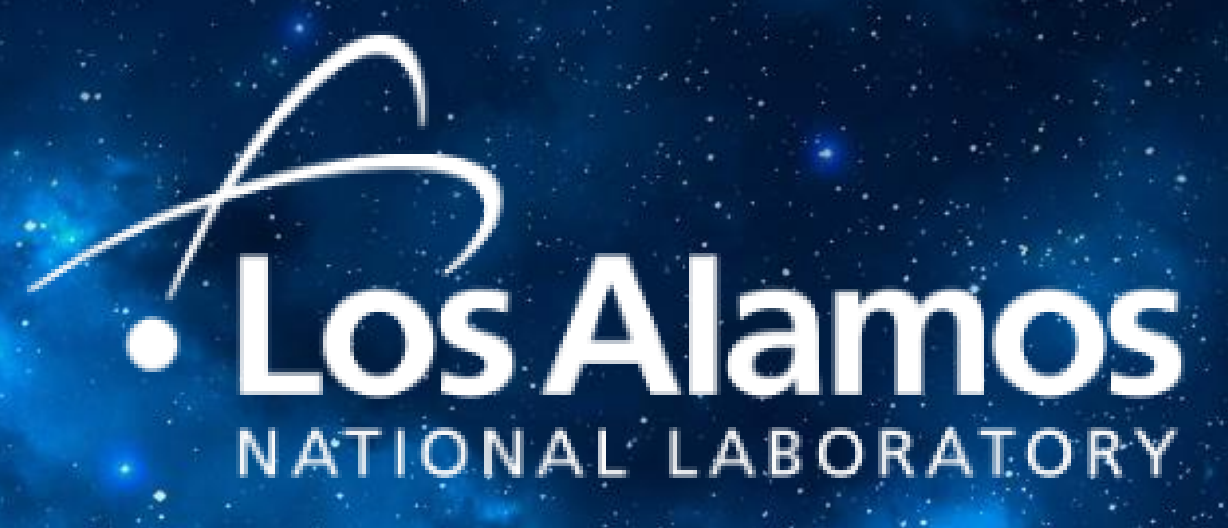

EST. 1943
BLUE URIGIN

$$
\text { SPACEK }
$$

ㄷㄴㄷ1ட

AEROJET ROCKETDYNE

Raytheon

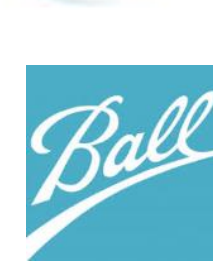
Orbital ATK 8 Lonze

$\therefore \because$ $\because \because \therefore$ SPACE TANGO $\because \because \bullet$

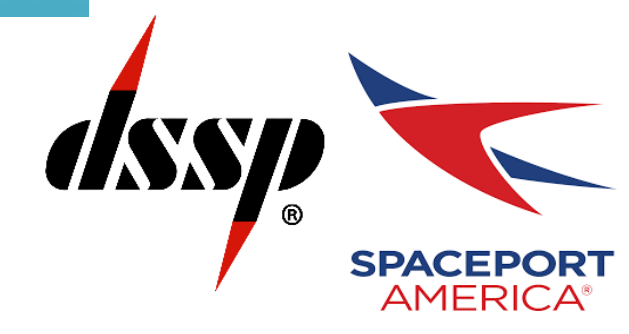

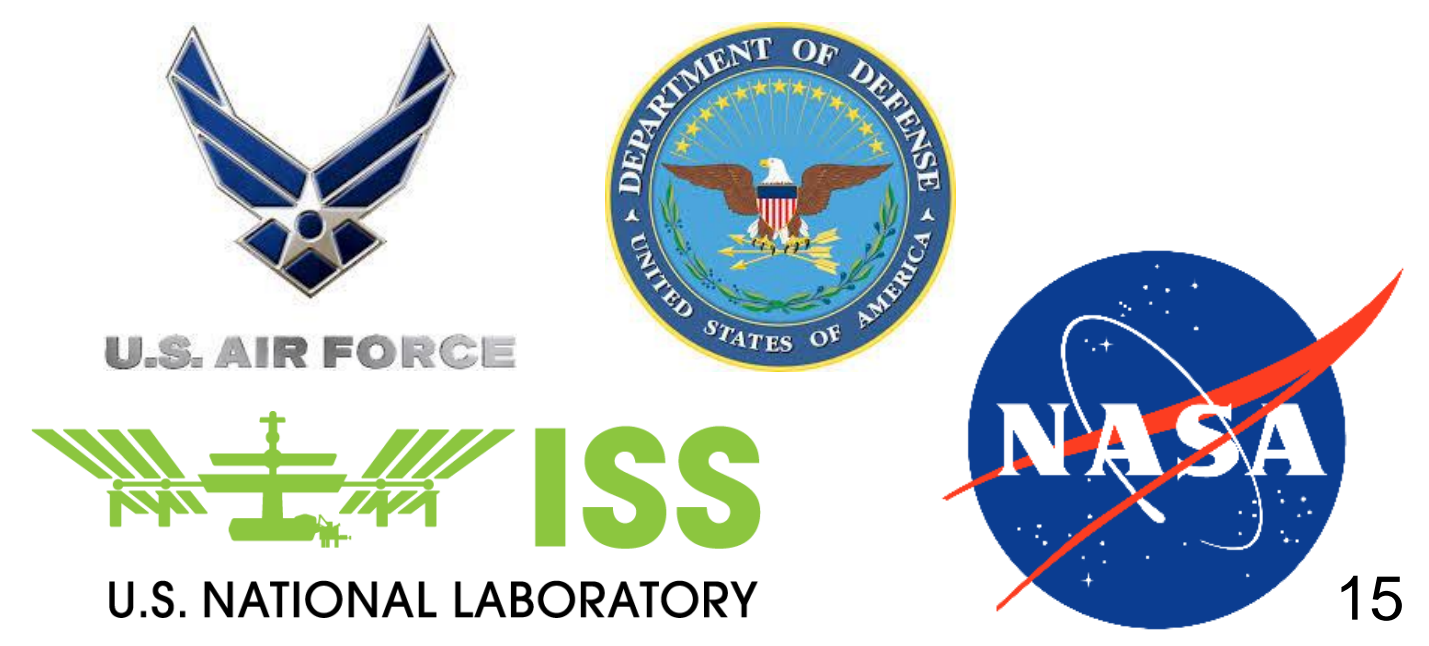




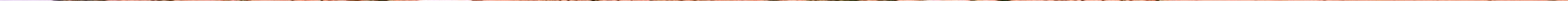




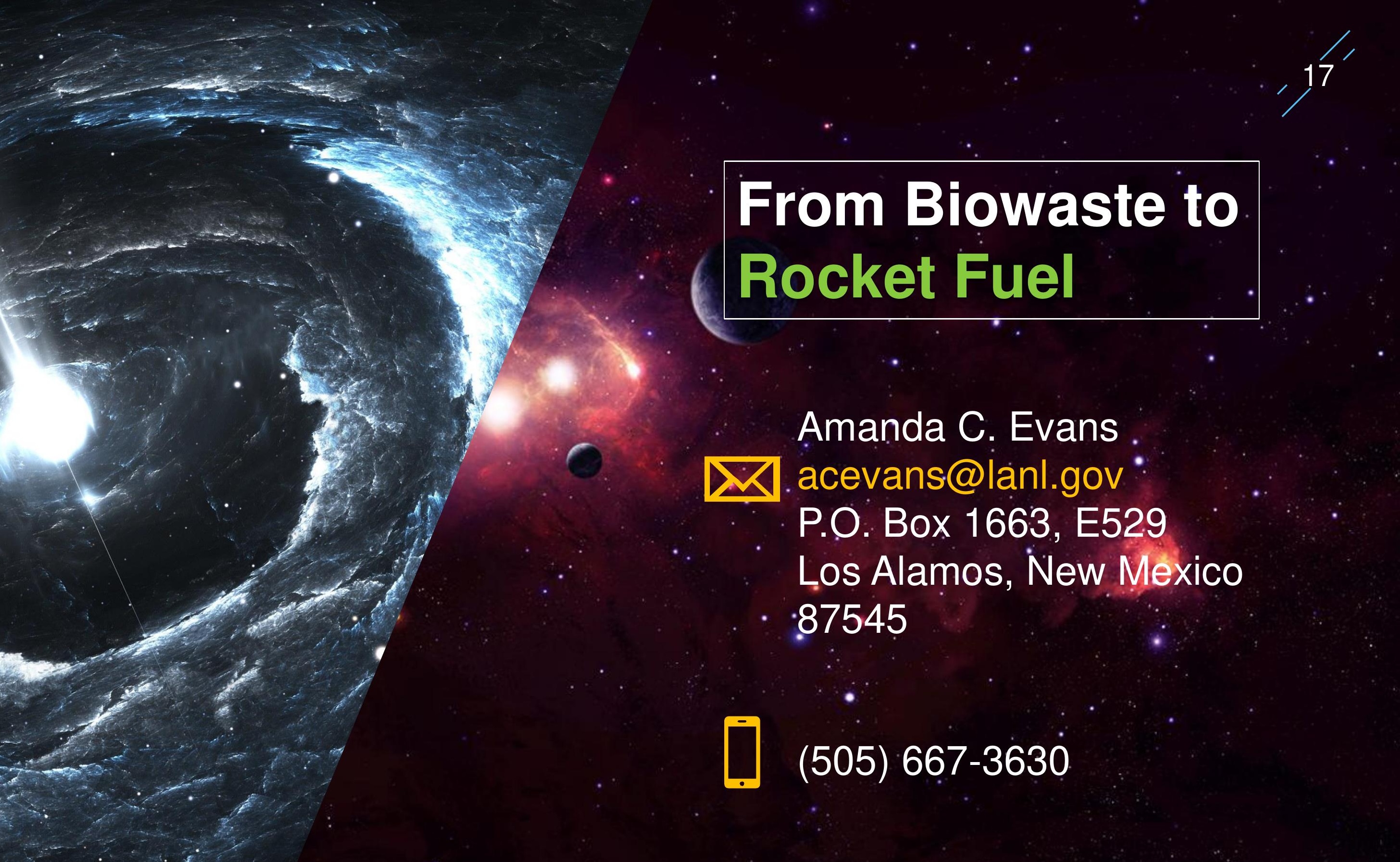

